\title{
Recovery potential of transplanted oligoprogenitor cells derived from human dental pulp stem cells in Lysophosphatidyl choline demyelination model
}

\author{
Hamidabadi $\mathrm{HG}^{1}$, Bojnordi $\mathrm{MN}^{1}$, Ehsani $\mathrm{S}^{2}$ \\ Immunogenetic Research Center, Faculty of Medicine, Mazandaran University of Medical Sciences, \\ Sari, Iran. bojnordi@modares.ac.ir
}

\begin{abstract}
AIM: Here we used a demyelination model using an injection of Lysophosphatidylcholine )LPC( in the corpus collosum to examine the myelination activity of differentiated oligodendrocytes derived from Human dental pulp stem cells )hDPDSCs( according to a two step induction protocol.

METHODS AND MATERIALS: The cells were cultured in DMEM-F12 medium containing 1M Retinoic acid and were treated with $5 \mathrm{ng} / \mathrm{ml}$ platelet-derived growth factor, $10 \mathrm{ng} / \mathrm{ml}$ basic fibroblast growth factor for 8-10 days. The differentiation cells were examined via the expression of specific glial markers: Olig2 and O4. Cells were transplanted in to a demylinated rat corpus callosum. The alteration of the demyelination extension as well as remyelination intensity was examined via a specific myelin staining: Luxol Fast Blue and immunohistochemistry.

RESULTS: Differentiated oligoprogenitor cells were confirmed via immunofluorescence staining with specific glial markers: Olig2 and O4. Also, the amount of demyelination was decreased and intensity of remyelination showed an increase after an engraftment of differentiated cells. Immunohistochemistry for evaluation of PLP expression proved the mature myelinating oligodendrocytes.

CONCLUSION: hDPSCs can be induced to transdifferentiate into oligoprogenitor cells and respond to the routinely applied regents for glial differentiation of Mesenchymal stem cells. hDPSCs may be a valuable source for cell therapy in neurodegenerative diseases (Fig. 4, Ref. 30). Text in PDF www.elis.sk KEY WORDS: dental pulp stem cells, lysophosphatidylcholine, corpus callosum, oligodendrocyte, differentiation.
\end{abstract}

\section{Introduction}

The disruption in myelin sheath surrounding the fibres in central nervous system, leads to demyelination diseases such as: Multiple Sclerosis recognized with severe neurological symptoms, sensory and motor disabilities (Blakemore et al, 2007). Since the oligodendrocytes form the myelin sheet, transplantation of stem cell derived from oligodendrocytes can be used as an appropriate alternative therapy for the improvement of the remyelination in the demyelinated neural tissues (Blakemore et al, 2007).

Among various sources for cell therapy, dental pulp mesenchymal cells (hDPSCs) are considered a reliable source that can differentiate to neuroglia (Gronthos et al, 2002, Haratizadeh et al, 2016). Some special potentials include: low immunogenicity, antiinflammatory and multipotency, suggesting DPSCs for therapeutic

${ }^{1}$ Immunogenetic Research Center, Faculty of Medicine, Mazandaran University of Medical Sciences, Sari, Iran, and ${ }^{2}$ Department of Physiology, School of Medicine, Mazandaran University of Medical Sciences, Sari, Iran

Address for correspondence: M.N. Bojnordi, Immunogenetic Research Center, Faculty of Medicine, Mazandaran University of Medical Sciences, Sari, Iran. P.O. Box: 48471-91971.

Acknowledgement: The study was supported by the research grant from Mazandaran University of Medical Sciences, Sari, Iran (No.2275). strategies in regenerative medicine (Gronthos et al, 2002, Mojaverrostami et al, 2018). hDPSCs have the differentiation capacity to oligoprogenitor cells and mature oligodendrocytes (Gronthos et al, 2002, Crawford et al, 2017, Sanen et al, 2017). But the functionality of glial cells derived from hDPSCs has been still unclear. To this aim, induction of an animal demyelination model can prepare the efficient environment to examine the functionality of differentiated oligodendrocytes from hDPSCs. Injection of LPC into the corpus callosum of the focal demyelinated area provides the possibility for monitoring the maturation of transplanted oligoprogenitor via their myelinogenic activity (Martens et al, 2014, Lambrichts et al, 2017, Ullah et al, 2017). In this study, we differentiated hDPSCs to the oligoprogenitor cells and transplanted the cells to a demyelinated corpus callosum using an injection of LPC to evaluate the maturation of the transplanted cells. This study might be considered as a new therapeutic strategy for demyelinating disorders such as Multiple sclerosis (MS).

\section{Materials and methods}

\section{Extraction and culture of hDPSCs}

hDPSCs were isolated from Human dental pulp of molar teeth in Mazandaran University of Medical Sciences. After mechanical 
and enzymatic digestion with trypsin $0.25 \%$ (Gibco, USA) digestion, the tissues were centrifuged and the supernatant was removed and cultured in DMEM/F12 (Gibco, USA) medium supplemented with $15 \%$ FBS (Gibco, USA), streptomycin/penicillin, and Lglutamine (Gibco, USA).

Morphology and cell proliferation of the cultured cells were monitored daily via a phase contrast microscopy.

\section{Multipotency of hDPSCs}

Multilineage differentiation of hDPSCs was investigated by adipogenic and osteogenic differentiation via Oil Red $\mathrm{O}$ and Alizarin Red staining.

\section{Flow cytometry}

The mesenchymal stem cell markers: CD44, CD90, CD105 as well as CD34 and CD45, hematopoietic markers were tested using flow cytometry technique.

\section{Glial induction of hDPSCs}

Glial induction was done according to a two step induction protocol. Briefly, hDPSc at 4th passage was cultured in the presence of DMEM-F12 medium containing 1M Retinoic acid (Sigma Aldrich) for 4 days. The cells were treated with $5 \mathrm{ng} / \mathrm{ml}$ plateletderived growth factor (Sigma Aldrich), $10 \mathrm{ng} / \mathrm{ml}$ basic fibroblast growth factor (Sigma Aldrich) for 8-10 days.

\section{Immunocytochemistry analysis}

The glial characteristics of differentiated cells was confirmed using evaluation of glial specific markers, Olig2 and O4. Briefly, cells were fixed in $4 \%$ paraformaldehyde and were permeabilized with $0.2 \%$ Triton X-100 and washed with PBS. After blocking with $10 \%$ goat serum, the cells were incubated with primary antibodies: Mouse anti olig2 monoclonal antibody (abcam) and mouse anti-O4 monoclonal antibody (abcam). The next day, cells were washed and were exposed to a secondary antibody for 1 hour at the room temperature. Following washing and mounting with 4 , 6-diamidino-2-phenylindole (DAPI) the images were captured with an Olympus phase.

\section{Induction of LPC demyelination model}

Demyelination model was induced using a stereotaxic injection of $2 \mu \mathrm{l}$ of LPC ( $1 \%$, Sigma) into the corpus callosum of female Sprague-Dawley rats (Mozafari et al, 2010).

A demyelination area appeared after one week. Two groups were designed: The experiment group $(n=5)$ in which differentiated oligoprogenitor cells $(200,000$ cells) were injected directly into the corpus callosum using a Hamilton syringe connected to a 30-gauge needle and the control group, which received only $2 \mu \mathrm{l}$ of medium, without any cells $(n=5)$ (Pouya et al, 2010).

Labelling of the transplanted cells was done using the carbocyanine dye Cell Tra- cker TM CM-DiI (Life Technologies). The injection of the cells was done via a Hamilton syringe connected to a needle. Each experiment was repeated three times.

\section{Histological assessment for myelination alteration}

The LPC injected animals were sacrificed 1 week post injection of gliotoxin to confirm the demyelination model. Also, 2 weeks after the cell therapy, the demyelination and remyelination changes were examined using a specific myelin staining e.g. Luxol Fast Blue. The amount of demyelination and intensity of remyelination was calculated using a light microscopy and quantified via Image $\mathrm{J}$ software.

\section{Immunohistochemistry evaluation}

To trace the labelled cells, immunohistochemistry evaluation of PLP expression was performed for specific mature myelinating oligodendrocytes. Firstly, after the deparaffinization and rehydration, they were blocked with $10 \%$ goat serum and then they were incubated with anti-PLP overnight. The slides were incubated with a secondary antibody (goat antirat IgG, Abcam, UK) for $1 \mathrm{~h}$ at room temperature and then washed with PBS. The extension of demyelination and remyelination intensity of PLP staining was evaluated and quantified.

\section{Statistical analysis}

The statistical analysis was done with SPSS 13.0 software and data were analysed via one-way analysis of variance (ANOVA), followed by Tukey post hoc test ( $\mathrm{p}<0.05$ was considered significant).

\section{Results}

\section{Morphological Characteristics of hDPSCs}

The adherent potential of the isolated hDPSCs was monitored. The cultured cells exhibited a fibroblastic morphology that proved the mesenchymal characteristics of hDPSCs. They showed a high proliferation activity and were attached to the flask bottom (Fig. 1a).

The immune-positive reactivity to mesenchymal markers: $C D$ 44, CD 90, CD 105 was confirmed via flow cytometry. hDPSCs showed a negative tendency to hematopoietic markers CD34, CD45 (Moayeri A et al, 2017).
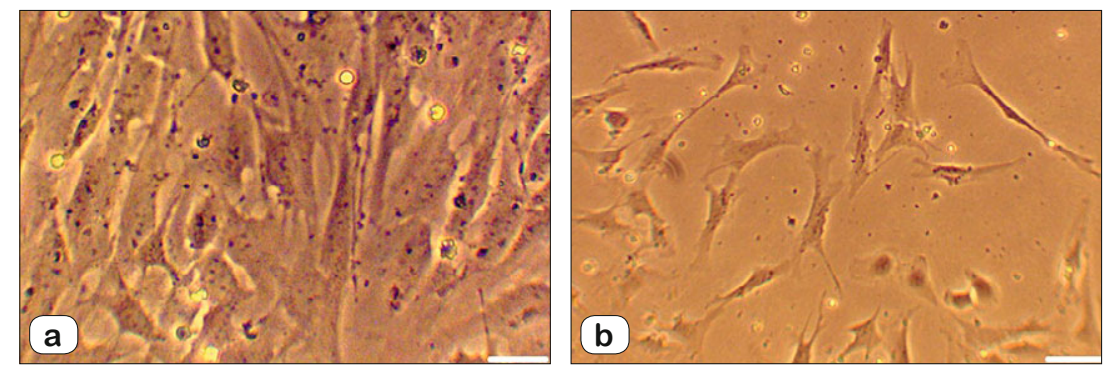

Fig. 1. The morphological phenotype of cultured hDPSCs. Cultured hDPSCs at the fourth passage (a). Oligoprogenitor cells derived from hDPSCs (b). Scale bars $10 \mu \mathrm{m}$. 

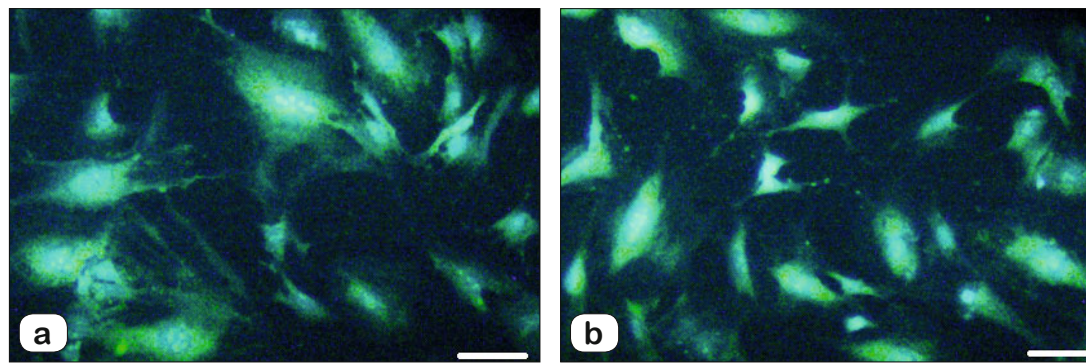

Fig. 2. Immunofluorescence staining of oligoprogenitor cells derived from hDPSCs immunepositive cells to oligo2 (a) and 04 (b) antibodies. Scale bars $10 \mu \mathrm{m}$.
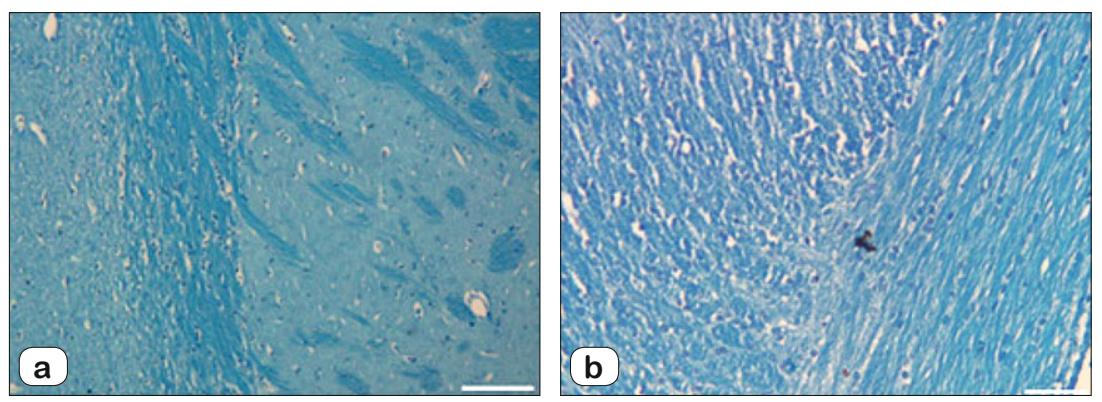

c

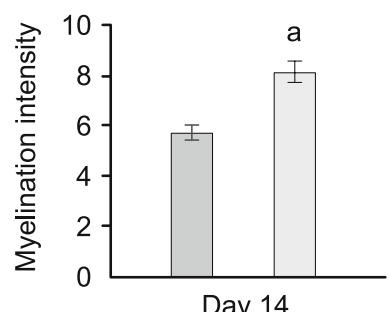

Day 14

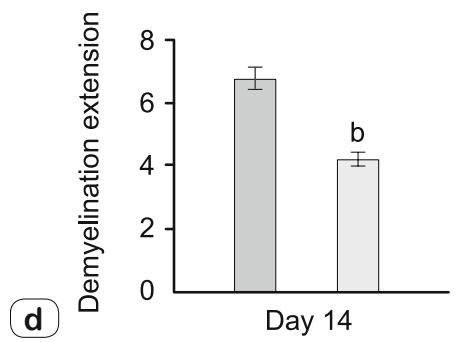

Fig. 3. The alteration of demyelination using the Luxol Fast Blue staining. Demyelinated corpus callosum induced one week post injection of LPC toxin (a) and 2 weeks post cell transplantation(b). (c) Compression of quantitative data for the remyelination intensity and demyelination extension in the cell transplanted group compared to the control group (d). Scale bars $=60 \mu \mathrm{m}$. a: significant increase in the control group, b: a significant decrease with control group.

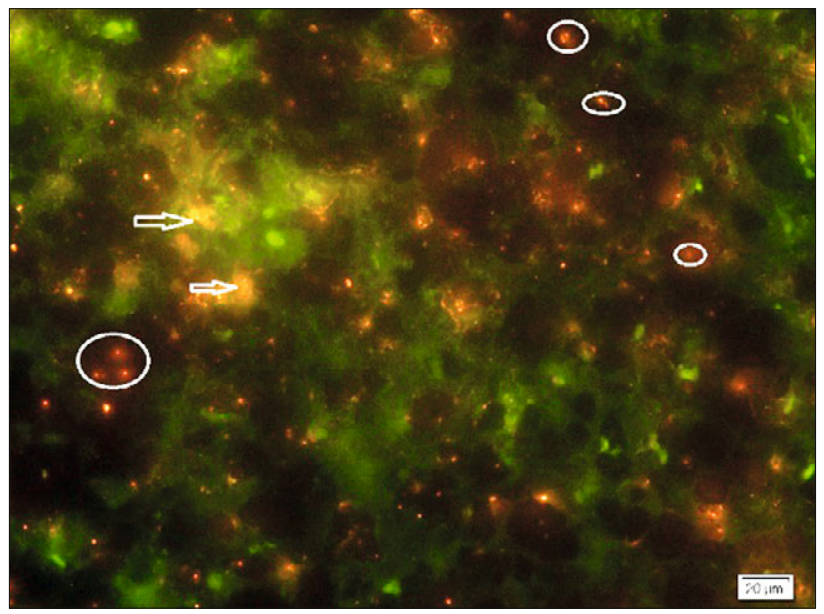

Fig. 4. Immunostaining of cell transplanted groups. Oligoprogenitor like cells labelled with DiI (red, indicated by arrows) showed PLP immunoreactivity (yellow, circles) Scale bars $=20 \mu \mathrm{m}$.
Oil Red staining proved the Adipogenic differentiation of hDPSCs and the formation of calcium deposits was detected using the Alizarin Red staining (Moayeri A et al, 2017).

Derivation of oligoprogenitor cells from hDPSCs

Morphological changes of the differentiated cells were confirmed via a phase contrast microscopy (Fig. 1a). After exposure to inducers, hDPSCs exhibited a phenotype resembling an oligodendrocyte. Fibroblastic hDPSCs converted to differentiated bipolar or branched cells (Fig. 1b).

The immunostaining of differentiated oligoprogenitor cells from hDPSCs

Also, the differentiated oligoprogenitor cells were confirmed via immunofluorescent staining with specific glial markers: Olig2 and $\mathrm{O} 4$ (Fig. 2a, b). The mean percentages of positive cells for these glial markers were $42.7 \pm 5.14 \%$ and $46.34 \pm 9.05 \%$.

\section{LPC demyelination model}

Demyelination was induced using a stereotaxic injection of LPC into the rat corpus callosum. A significant local demyelinated area was detected using a histological evaluation, approximately after one week post toxin injection. Luxol Fast Blue staining was used for evaluation of amount of demyelination and remyelination intensity. The alteration of remyelination was monitored following 2 weeks post cells engraftment and was compared to the control group (Fig. 3).

\section{PLP immunohistochemistry}

PLP immunostaining proved the homing potential of the transplanted labelled differentiated cells within the corpus callosum. Most of these cells were PLP+, which is the marker of mature oligodendrocyte with a myelination activity (Fig. 4). The intensity of PLP staining was significantly higher in the cell transplanted group compared to the control group.

\section{Discussion}

Demyelinating disease such as MS leads to axonal loss, severe neurological symptoms, and a progressive disability. Stem cell therapy has been considered an alternative therapeutic strategy for demyelination diseases (Dai et al, 2013, Nazem Ghasemi et al, 2017, Bojnordi et al, 2016). hDPSCs is a reliable supply for generation of glial cells with no ethical limitation or tumorigenic 
activity (Dissanayaka et al, 2016, Wislet-Gendebien et al, 2005, Nazm Bojnordi et al, 2016).

In this study, oligoprogenitor cells derived from hDPSCs were examined to increase the remyelination in a toxin model of the corpus callosum. hDPSCs were cultured and treated with neurogenic inducers such as: Retinoic acid, EGF and bFGF.

The morphological dates showed that hDPSCs appeared with fibroblastic morphology, similar to the previous reports (Bojnordi et al, 2018, Sher et al, 2008, Luessi et al, 2014).

At the end stage of treatment, some morphological changes appeared as differentiated oligoprogenitor cells were detected with a multipolar morphology.

Similar reports confirmed the differentiation of hDPSCs to glial phenotype in the presence of specific inducers (Gronthos et al, 2000, Lambrichts et al, 2017, Schmalz et al, 2014).

The specific cocktail including the essential components includes: Retinoic acid, growth factors and cytokines triggers a positive alteration in the specific glial gene expression and mediates a glial differentiation of hDPSCs using an activation of myelinogenic genes and proteins. We found out, that the differentiated oligoprogenitor cells completed the maturation process following the engraftments into the LPC demyelination model. Dates of immunohistochemistry confirmed the maturation of oligoprogentor cells using a staining with specific markers: PLP. Remyelination activity of transplanted oligoprogenitor cells was confirmed using a myelin specific staining: Luxol Fast Blue that can show a myelination alteration post cell transplantation. Remyelination was augmented following engraftment of the oligoprogenitor cells into the LPC demyelination model. Furthermore, the advantage of such demyelination model is in approving the validity of maturation of oligoprogenitor cells into functional and mature myelinating oligodendrocytes.

These findings are similar to the previous results that confirmed the significant remyelination in LPC demyelination models of the brain and the spinal cord after transplantation of the glial cells derived from Embryonic stem cells and Bone marrow stromal stem cells (Jaramillo-Merchan, 2013, Hu and Zhan, 2009, Tirotta et al, 2010, Narkilahti et al, 2009, Pouya et al, 2011, Alizadeh et al, 2017).

It is concluded that the transplantation of differentiated glial cells is considered as the therapeutic strategy with an advantage for remyelination in demyelinating diseases including MS.

\section{References}

1. Aditya GS, Chakrabarty A. Multiple sclerosis and demyelination. Curr Diagn Pathol 2007; 13: 193-202.

2. Alizadeh R, Hassanzadeh G, Joghataei MT, Soleimani M, Moradi F, Mohammadpour S et al. In vitro differentiation of neural stem cells derived from human olfactory bulb into dopaminergic-like neurons. Eur J Neurosci 2017; 45: 773-784.

3. Blakemore WF. Regeneration and repair in multiple sclerosis: the view of experimental pathology. J Neurol Sci 2008; 15: 265: 1-4.
4. Bojnordi MN, Movahedin M, Tiraihi T, Javan M.A. simple co-culture system for generation of embryonic stem-like cells from testis. Iranian Red Crescent Med J 2012; 14 (12): 811.

5. Crawford AH, Stockley JH, Tripathi RB et al. Oligodendrocyte progenitors: adult stem cells of the central nervous system. Exp Neurol 2014; 260: $50-55$.

6. Dai LG, Huang GS, Hsu SH. Sciatic nerve regeneration by cocultured Schwann cells and stem cells on microporous nerve conduits. Cell Transplant 2013; 22 (11): 2029-2039.

7. Dissanayaka WL, Hargreaves KM, Jin L, Samaranayake LP, Zhang C. The interplay of dental pulp stem cells and endothelial cells in an injectable peptide hydrogel on angiogenesis and pulp regeneration in vivo. Tissue Eng Part A 2015; 21 (3-4): 550-563.

8. Ghasemi N, Razavi S, Mardani M, Esfandiari E, Salehi H, Zarkesh Esfahani SH. Transplantation of human adipose-derived stem cells enhances remyelination in lysolecithin-induced focal demyelination of rat spinal cord. Mol Biotechnol 2014; 56 (5): 470-478.

9. Schmalz G, Smith AJ. Pulp Development, Repair, and Regeneration: Challenges of the Transition from Traditional Dentistry to Biologically Based Therapies. J Endodontics 2014; 40 (4 Supplement): S2-S5

10. Gronthos S, Brahim J, Li W, Fisher LW, Cherman N, Boyde A et al. Stem cell properties of human dental pulp stem cells. J Dent Res 2002; 81 (8): 531-535.

11. Gronthos S, Mankani M, Brahim J, Robey PG, Shi S. Postnatal human dental pulp stem cells (DPSCs) in vitro and in vivo. Proc Nat Acad Sci 2000; 97 (25): 13625-13630.

12. Haratizadeh S, Bojnordi MN, Niapour A, Bakhtiari M, Hamidabadi HG. Improvement of neuroglial differentiation from human dental pulp stem cells using CSF. J Mazandaran University Med Sci 2016; 26: $1-14$.

13. Hu BY, Zhang SC. Differentiation of spinal motor neurons from pluripotent human stem cells. Nat Protoc 2009; 4: 1295-1304.

14. Jaramillo-Merchan J, Jones J, Ivorra JL, Pastor D, Viso-Leon MC, Armengol JA, Molto MD, Geijo-Barrientos E, Martinez S. Mesenchymal stromal-cell transplantsinduce oligodendrocyte progenitor migration and remyelination in a chronic demyelinationmodel. Cell Death Dis 2013; 4: e 779 .

15. Tirotta E, Carbajal KS, Schaumburg CS, Whitman L, Lane TE. Cell replacement therapies to promote remyelination in a viral model of demyelination. J Neuroimmunol 2010; 224: 101-107.

16. Lambrichts I, Driesen RB, Dillen Y, Gervois P, Ratajczak J, Vangansewinkel T, Wolfs E, Bronckaers A, Hilkens P. Dental Pulp Stem Cells: Their Potential in Reinnervation and Angiogenesis by Using Scaffolds. J Endod 2017; 43 (9S): S12-S16.

17. Luessi F, KuhlmannT, Zipp F. Remyelinating strategies in multiple sclerosis. Expert Rev Neurother 2014; 14: 1315-1334.

18. Martens W, Sanen K, Georgiou M, Struys T, Bronckaers A, Ameloot M, Phillips J, Lambrichts I. Human dental pulp stem cells can differentiate into Schwann cells and promote and guide neurite outgrowth in an aligned tissue-engineered collagen construct in vitro. FASEB J 2014; 28 (4): 1634-1643.

19. Mojaverrostami S, Bojnordi MN, Ghasemi-Kasman M, Ebrahimzadeh MA, Hamidabadi HG. A review of herbal therapy in multiple sclerosis. Adv Pharm Bull 2018; 8 (4): 575-590. 
20. Mozafari S, Sherafat MA, Javan M, Mirnajafi-Zadeh J, Tiraihi T. Visual evoked potentials and MBP gene expression implyendogenous myelin repair in adult rat optic nerve and chiasma following local lysolecithin induced demyelination. Brain Res 2010; 1351: 50-56.

21. Narkilahti S, Hovatta O, Elovaara I. Stem cells in therapy of multiple sclerosis. Duodecim 2009; 125: 965-973.

22. Nazm Bojnordi M, Movahedin M, Tiraihi T, Javan M. Alteration in genes expression patterns during in vitro differentiation of mouse spermatogonial cells into neuroepithelial-like cells. Cytotechnology 2013; 65: 97-104.

23. Pouya A, Satarian L, Kiani S, Javan M, Baharvand H. Human induced pluripotent stem cells differentiation into oligodendrocyte progenitors and transplantation in a rat model of optic chiasm demyelination. PLoS One 2011; 6: e27925.

24. Sanen K, Martens W, Georgiou M, Ameloot M, Lambrichts I, Phillips J. Engineered neural tissue with Schwann cell differentiated human dental pulp stem cells: potential for peripheral nerve repair? J Tissue Eng Regen Med 2017; 11 (12): 3362-3372.

25. Sher F, Balasubramaniyan V, Boddeke E, Copray S. Oligodendrocyte differentiation and implantation: new insights for remyelinating cell therapy. Curr Opin Neurobiol 2008; 21: 607-607.
26. Bojnordi MN, Ebrahimi-Barough S, Vojoudi E, Hamidabadi HG. Silk nanofibrous electrospun scaffold enhances differentiation of embryonic stem like cells derived from testis in to mature neuron. J Biomed Mater Resa Part A 2018; 106 (10): 2662-2669.

27. Ullah I, Park JM, Kang YH, Byun JH, Kim DG, Kim JH, Kang DH, Rho GJ, Park BW. Transplantation of Human Dental Pulp-Derived Stem Cells or Differentiated Neuronal Cells from Human Dental Pulp-Derived Stem Cells Identically Enhances Regeneration of the Injured Peripheral Nerve. Stem Cells Dev 2017; 26 (17): 1247-1257.

28. Kotter MR, Setzu A, Sim FJ, Van Rooijen N, Franklin RJ. Macrophage depletion impairs oligodendrocyte remyelination following lysolecithin-induced demyelination. Glia 2001; 35: 204-212.

29. Wislet-Gendebien S, Hans G, Leprince P et al. Plasticity of cultured mesenchymal stem cells: switch from nestin-positive to excitable neuronlike phenotype. Stem Cells 2005; 23: 392-402.

30. Wislet-Gendebien S, Hans G, Leprince P, Rigo JM, Moonen G, Rogister B. Plasticity of cultured mesenchymal stem cells: switch from nestin-positive to excitable neuronlike phenotype. Stem Cells 2005; 23 (3): $392-402$.

Received March 9, 2021. Accepted March 16, 2021. 